Rev. salud pública. 10 (4):650-657, 2008

\title{
Staphylococcus aureus en Residentes de un Hogar de Ancianos de Cartagena
}

\author{
Staphylococcus aureus in residents from a nursing-home in \\ Cartagena \\ Alfonso Bettin, Paola Suárez, Andrés Bedoya y Niradiz Reyes
}

Facultad de Medicina, Universidad de Cartagena. Colombia. alfonsobettin@yahoo.es; paolasuarezalvarez@gmail.com; andresbo2003@yahoo.es; niradiz@gmail.com

Recibido 3 Marzo 2008/Enviado para Modificación 23 Agosto 2008/Aceptado 25 Agosto 2008

\section{RESUMEN}

Objetivos Determinar la prevalencia de colonización nasal de Staphylococcus aureus, la susceptibilidad a antibióticos de los aislamientos y su posible asociación con factores de riesgo, en los residentes del Hogar Asilo de Ancianos San Pedro Claver de la ciudad de Cartagena de Indias durante el segundo semestre del año 2007.

Métodos Con el debido consentimiento informado, para cada sujeto participante se tomaron hisopados nasales por una única vez durante el estudio. Se identificaron cepas de Staphylococcus aureus usando métodos clásicos y se determinó la susceptibilidad a antibióticos de los aislamientos mediante el método de difusión por disco según los estándares del CLSI. La información recolectada de las historias clínicas y con ayuda de un cuestionario se utilizó para analizar la asociación con factores de riesgo potenciales usando el programa SPSS 13.0 para Windows.

Resultados Se obtuvieron 11 aislamientos positivos para Staphylococcus aureus, de un total de 69 individuos participantes, lo que correspondió a una prevalencia de 15,9 $\%$. No se detectaron cepas resistentes a meticilina. La portación nasal de Staphylococcus aureus estuvo asociada significativamente con limitaciones en el nivel de movilidad y con la presencia de lesiones cutáneas. No se encontró asociación significativa con otros de los diferentes factores de riesgo analizados.

Conclusiones. La portación nasal de S. aureus encontrada en este estudio es mucho más baja que las reportadas en estudios similares en Colombia y otros países, teniendo en cuenta que la población estudio es vulnerable a la colonización con este patógeno.

Palabras Clave: Portador sano, Staphylococcus aureus, resistencia a la Meticilina (fuente: DeCS, BIREME).

\section{ABSTRACT}

Objectives Determining Staphylococcus aureus nasal carriage, antibiotic susceptibility and association with potential risk factors in residents from the Hogar Asilo de Ancianos San Pedro Claver nursing-home in Cartagena during the second semester of 2007. 
Methods Nasal swabs were taken from each person participating in the study after they had signed an informed consent form. Staphylococcus aureus strains were identified by classical methods; antibiotic susceptibility was determined by disk diffusion methods, according to CLSI standards. SPSS for Windows 13.0 statistical package was used for analysing data collected from medical records and from a questionnaire for analysing association with potential risk factors.

Results 11 Staphylococcus aureus isolates were obtained from 69 participants, corresponding to $15.9 \%$ prevalence. No methicillin-resistant strains were detected. Staphylococcus aureus nasal carriage was significantly associated with limited mobility and skin lesions. There was no significant association with the other risk factors analysed.

Conclusions Staphylococcus aureus nasal carriage found in this study was lower than that reported from other similar studies in other countries, taking into account that this is a population at risk for colonisation by this pathogen.

Key Words: Carrier state, Staphylococcus aureus, methicillin resistance (source: $\mathrm{MeSH}, \mathrm{NLM}$ ).

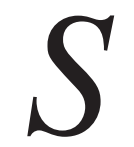

taphylococcus aureus (S. aureus) es un importante patógeno humano causante de enfermedades intra- y extra- hospitalarias, debido a su amplia y frecuente distribución. Las infecciones estafilocócicas muchas veces están asociadas a infecciones de piel y tejidos blandos, e incluyen síndromes que van desde una baja morbilidad y mortalidad, tales como foliculitis e intoxicaciones alimentarias, hasta lesiones sistémicas fatales, tales como endocarditis y síndrome de shock toxico, entre otras (1).

El nicho ecológico principal del S. aureus en humanos lo constituyen las fosas nasales anteriores, reconocidas como una fuente potencial de infección y factor de riesgo elevado para subsecuentes infecciones invasivas (2-4). Se ha reconocido que muchas de las infecciones por $S$. aureus ocurren en personas que están colonizadas con la bacteria $(4,5)$, desconociéndose las razones por las que la bacteria coloniza solo del 30 al $40 \%$ de los individuos en la población general. De acuerdo con la población estudiada, se reportan variaciones en la prevalencia e incidencia de la portación nasal de $S$. aureus. En la revisión realizada por Kluytmans et al, en 1997 (6), se muestra que la prevalencia de portación en el personal hospitalario varia entre $16,8 \%$ a $56,1 \%$, en pacientes ambulatorios entre $10,2 \%$ a $85,0 \%$ y en pacientes hospitalizados entre $14,3 \%$ a $52,5 \%$. Como caso nacional tenemos un estudio realizado en la clínica Universitaria Bolivariana, Medellín, en donde se halló un alto porcentaje (85\%) de aislamientos de Staphylococcus aureus en fosas nasales del personal de la unidad de terapia intensiva (7). 
En poblaciones donde prevalecen las condiciones de hacinamiento, tales como escuelas de medicina, guarderías, prisiones y hogares de atención del adulto mayor, se favorece la colonización por $S$. aureus. Estas poblaciones vulnerables a la colonización e infección por esta bacteria, han sido objeto de estudios para establecer la tasa de portación de $S$. aureus, con el fin de fortalecer o implementar nuevas medidas de control, para prevenir brotes de enfermedades asociadas a este patógeno (8-12).

Una de estas poblaciones altamente vulnerables corresponde a los adultos mayores, que conviven en hogares asilos en los que el riesgo de colonización por S. aureus podría estar asociado con el incremento en la debilidad, limitaciones en la movilidad física y la presencia de tratamientos invasivos o lesiones (13). Como lo demostró un estudio en Turquía, en un grupo de 79 ancianos voluntarios residentes de un hogar asilo, la tasa de portación nasal de $S$. aureus fue de $43 \%$ y estuvo asociada a lesiones en piel, hospitalización previa y uso de antibióticos en los últimos seis meses (14). Mientras que en Alemania, en otro estudio realizado en tres hogares asilo, la tasa de portación nasal fue de 36,6 \%, encontrándose como factores de riesgo significativos la diabetes mellitus, la hipertensión, terapia antibiótica previa, y nivel de atención tipo dos (11).

Estos estudios en general sugieren que existen ciertos factores de riesgo implicados en la portación y diseminación de $S$. aureus, lo que hace necesario analizar la asociación de otros factores y realizar estudios en diferentes áreas geográficas de nuestro país para conocer la situación global de portación de este patógeno en comunidades en riesgo, como la de residentes de asilos de ancianos. Por lo tanto, el objetivo de este estudio fue determinar la prevalencia de portadores nasales y analizar asociaciones con posibles factores de riesgo en los residentes del asilo San Pedro Claver, de la Ciudad de Cartagena de Indias, para así, de acuerdo con los resultados obtenidos, fortalecer las medidas de control, para prevenir futuras enfermedades en la población de estudio, particularmente expuesta a infecciones ocasionadas por S. aureus.

\section{MÉTODOS}

Diseño y Población de estudio

El presente fue un estudio descriptivo de corte transversal en el cual se determinó la prevalencia de portadores nasales de Staphylococcus aureus en la población estudio, en el periodo comprendido entre Septiembre a Diciembre de 2007. El diseño metodológico propuesto fue de tipo observacional y la población estudio estuvo constituida por los residentes del hogar asilo de ancianos San Pedro 
Claver, que estuvieron de acuerdo en participar de este proyecto, los cuales firmaron un consentimiento informado para participar en la investigación. Para aquellos individuos con algún grado de discapacidad mental que les impidiera dar su consentimiento informado, se contó con el consentimiento informado del representante legal de la institución Hogar Asilo San Pedro Claver. El estudio contó con la aprobación del Comité de Ética de la Universidad de Cartagena. La información fue recolectada del propio participante, la historia clínica, el personal medico y los respectivos cuidadores, con lo cual se completó un cuestionario. Entre las variables incluidas estuvieron la edad, sexo, enfermedades (hipertensión, diabetes mellitus, enfermedad coronaria, enfermedad renal, cáncer, etc), lesiones en piel, hospitalizaciones y terapia antimicrobiana previa a tres y seis meses, movilidad (definida por: normal y limitada) y condiciones de residencia (número de camas por habitación, tiempo de residencia en el asilo).

Recolección de muestras y pruebas microbiológicas

Las muestras nasales fueron obtenidas de cada sujeto utilizando hisopos de algodón estériles. Cada hisopado de las fosas nasales se rotó tres veces en sentido de las manecillas del reloj y tres veces en sentido contrario (14). Los hisopos fueron colocados inmediatamente en medio de transporte de Stuart (OXOID, England), transportados en hielo $\left(4^{\circ} \mathrm{C}\right)$ y llevados hasta el laboratorio de Microbiología (Universidad de Cartagena), en donde se inocularon en placas de agar manitol sal (BBL, Becton, USA). Las placas fueron incubadas a $37^{\circ} \mathrm{C}$ por 48 horas, al término de las cuales se examinaron las características de las colonias (15). Todas las colonias manitol-sal positivas fueron subcultivadas en agar nutritivo (Difco, Michigan, USA) a $37^{\circ} \mathrm{C}$ por $24-48$ horas. Los aislamientos con morfología microscópica de cocos Gram positivos, que resultaron positivos a las pruebas de catalasa, coagulasa en tubo (BBL, Coagulase plasma Rabbit, Becton, USA), y de aglutinación en látex (Staphaurex, Remel, UK), fueron catalogados como Staphylococcus aureus. Los cultivos nasales negativos se reconfirmaron.

Pruebas de Susceptibilidad

La determinación de la sensibilidad antibiótica se realizó a través del método de difusión por disco según los estándares del Instituto de Estándares Clínicos y de Laboratorio (CLSI) (16). Los antibióticos evaluados fueron: rifampicina (5 $\mu \mathrm{g})$, clindamicina $(2 \mu \mathrm{g})$, eritromicina $(15 \mu \mathrm{g})$, gentamicina $(10 \mu \mathrm{g})$, vancomicina $(30 \mu \mathrm{g})$ y oxacilina $(1 \mu \mathrm{g})$. Como control se utilizó la cepa de $S$. aureus ATCC 25923. Después de 24 horas de incubación en aerobiosis a $35^{\circ} \mathrm{C}$, se determinó el diámetro de los halos de inhibición (15). 
Procesamiento de la información y análisis estadístico Con base en los resultados microbiológicos, los sujetos fueron divididos en dos grupos: Portadores y No Portadores, de acuerdo a si presentaron o no colonización nasal con S. aureus. Para la determinación de la tasa de portador nasal en la población estudio y su asociación con factores de riesgo se realizó análisis univariado con las variables independientes usando como medida estadística la prueba exacta de Fisher. Los análisis estadísticos fueron realizados con el programa SPSS 13.0 para Windows (SPSS Inc., Chicago, IL).

\section{RESULTADOS}

En el presente estudio participaron un total de 69 individuos, 34 hombres (49,3 $\%)$ y 35 mujeres $(50,7 \%)$, con una edad media de 76,37 $\pm 10,5$ años. Seis individuos adicionales rehusaron a la toma de muestras (tres hombres y tres mujeres). En el estudio se obtuvieron 11 aislamientos que dieron pruebas confirmatorias positivas para $S$. aureus, lo que correspondió a una prevalencia de $15,9 \%$. Las pruebas de susceptibilidad indicaron que todas las cepas aisladas fueron susceptibles a meticilina (MSSA) y al resto de los antibióticos ensayados (rifampicina, clindamicina, eritromicina, gentamicina y vancomicina); no se detectaron cepas resistentes a meticilina (MRSA). El análisis estadístico no encontró asociación significativa entre la portación nasal de la bacteria y las diferentes variables analizadas, excepto con el nivel de movilidad, ya que aquellos individuos con movilidad limitada tuvieron mas probabilidades de estar colonizados con MSSA (Tabla 1). La presencia de lesiones cutáneas estuvo asociada a la portación nasal de $S$. aureus en mujeres ( $p$ value $=0.040$, prueba exacta de Fisher). La Tabla 2 muestra las principales características de los casos portadores de MSSA.

Tabla 1. Comparación de los casos: portadores nasales de MSSA

\begin{tabular}{|c|c|c|c|}
\hline Parámetros & $\begin{array}{c}\text { MSSA }(+) \\
\mathrm{n}=11(15,9 \%)\end{array}$ & $\begin{array}{c}\text { MSSA }(-) \\
n=58(84,1 \%)\end{array}$ & $p$ value ${ }^{*}$ \\
\hline Sexo (Masculino/Femenino) & $5 \sqrt{6}$ & $29 / 29$ & NS \\
\hline Promedio de Edad (rango) & $74(48-93)$ & $76,4(46-97)$ & NS \\
\hline Diabetes mellitus & 0 & 5 & NS \\
\hline Hospitalizaciones-USM & 1 & 14 & NS \\
\hline Lesiones cutáneas (Si/No) & $5 / 6$ & $18 / 40$ & NS \\
\hline Uso de antibióticos-USM & 0 & 5 & NS \\
\hline $\begin{array}{l}\text { Movilidad } \\
\text { (Limitada/Normal) }\end{array}$ & $8 / 3$ & $17 / 36$ & 0.02 \\
\hline Hipertension (Si/No) & $2 / 9$ & $20 / 33$ & NS \\
\hline
\end{tabular}




\section{DISCUSION}

Las infecciones causadas por $S$. aureus son una causa significativa de morbilidad y mortalidad en adultos mayores $(17,18)$ y se ha establecido que un factor de riesgo significativo para el desarrollo de infecciones es la portación nasal previa de $S$. aureus $(4,5)$. Se ha considerado que la colonización de las fosas nasales es un proceso normal, pero que a su vez, también puede ser fuente de infección invasiva (5). Aunque la relación entre la colonización y el proceso infeccioso no está claramente definida, se ha establecido que existen factores intrínsecos tanto del huésped como de las cepas colonizadoras asociados tanto a la colonización como a la infección (5).

Tabla 2. Características de los casos portadores de MSSA

\begin{tabular}{|c|c|c|c|c|c|c|c|c|c|c|c|}
\hline \multirow{2}{*}{ Caracteristicas } & \multicolumn{11}{|c|}{ Individuo No. } \\
\hline & 1 & 2 & 3 & 4 & 5 & 6 & 7 & 8 & 9 & 10 & 11 \\
\hline Género & $M$ & $M$ & $M$ & $M$ & $M$ & $\mathrm{~F}$ & $F$ & $\mathrm{~F}$ & $\mathrm{~F}$ & $\mathrm{~F}$ & $\mathrm{~F}$ \\
\hline Edad & 74 & 72 & 75 & 74 & 68 & 93 & 48 & 86 & 60 & 87 & 77 \\
\hline Diabetes mellitus & No & No & No & No & No & No & No & No & No & No & No \\
\hline $\begin{array}{l}\text { Hospitalizaciones-USM } \\
\text { últimos seis meses) }\end{array}$ & $\mathrm{Si}$ & No & No & No & No & No & No & No & No & No & No \\
\hline Lesiones cutáneas & No & No & No & No & No & Si & $\mathrm{Si}$ & Si & Si & $\mathrm{Si}$ & No \\
\hline Uso de antibióticos-USM & No & No & No & No & No & No & No & No & No & No & No \\
\hline Nivel de movilidad & L & $N$ & L & L & L & L & $\mathrm{N}$ & $\mathrm{N}$ & L & $\mathrm{L}$ & L \\
\hline Hipertensión & No & No & No & $\mathrm{Si}$ & No & No & No & $\mathrm{Si}$ & No & No & No \\
\hline
\end{tabular}

* La presencia de lesiones cutáneas estuvo asociada a la portación nasal de $S$. aureus en mujeres (pvalue $=0.040$, prueba exacta de Fisher); Normal: N; Limitada: L; USM: Últimos seis meses

En el presente estudio encontramos que el 15,9\% de los residentes del asilo estuvieron colonizados con MSSA; no se encontraron portadores de MRSA. De hecho, las cepas aisladas fueron sensibles a todos los antibióticos ensayados: oxacilina, cefoxitina, vancomicina, gentamicina, eritromicina, clindamicina, y rifampicina. La prevalencia de portación nasal de $S$. aureus encontrada en este estudio es mucho más baja que las reportadas en estudios similares en otros países $(11,14,19,20)$. Una explicación a la baja prevalencia de $S$. aureus y a la susceptibilidad de las cepas encontradas a los diferentes antibióticos ensayados es que la deficiencia de recursos económicos y la baja cobertura médica de los residentes del asilo ha incidido en una reducción significativa en el uso de antibióticos, lo que a su vez ha reducido la presión selectiva de microorganismos resistentes, como ha sido sugerido en otros estudios $(11,21)$. Diversos estudios han encontrado asociación entre la portación nasal de $S$. aureus, tanto de cepas MSSA como MRSA, y diversos factores de riesgo en residentes de asilos de ancianos (10,11,14,19,21-23). En este estudio, encontramos que una movilidad limitada en los residentes del asilo y la presencia de lesiones cutáneas en el 
género femenino, representaron factores de riesgo significativos para la colonización por MSSA. Estos dos factores de riesgo asociados a la portación nasal de $S$. aureus han sido identificados previamente en diversos estudios en diferentes áreas geográficas del mundo $(10,11,14)$. En nuestro estudio encontramos una prevalencia de portación nasal de $S$. aureus menor a la reportada en otras áreas del mundo, y no detectamos cepas MRSA. Se ha establecido que tres mecanismos influyen en la prevalencia de MRSA en residentes de asilos de ancianos, siendo estos la selección de cepas resistentes a través del uso de antibióticos, la transmisión desde hospitales, y la contaminación cruzada en asilos (24). La población objeto del presente estudio se caracterizó por presentar una baja proporción de factores de riesgo previamente asociados a la prevalencia de colonización e infección por S. aureus, tales como una baja proporción de hospitalizaciones, un uso global de antibióticos bajo, y una baja prevalencia de enfermedades crónicas. Todas estas características de la población estudiada pudieron incidir positivamente en la baja prevalencia encontrada de portación nasal de la bacteria y a la ausencia de cepas MRSA $(5,11,14,23)$ *

Agradecimientos. A los estudiantes del semillero de investigación Sebimol y a los empleados, personal administrativo, y en especial a los residentes del asilo, por su valiosa colaboración para la realización de este estudio. Agradecimientos especiales a Ceyla Causil, estudiante de medicina y joven investigadora del grupo de investigación, por su valiosa participación en el estudio. Este trabajo fue financiado por la Universidad de Cartagena, Centro de Investigaciones Científicas y Tecnológicas, CICTE.

Conflicto de intereses: ninguno

\section{REFERENCIAS}

1. Lowy FD. Staphylococcus aureus infections. N Engl J Med 1998;339:520-32

2. Casewell MW. The nose: an underestimated source of Staphylococcus aureus causing wound infection. J Hosp Infect 1998;40 Suppl B:S3-11

3. Kuehnert MJ, Kruszon-Moran D, Hill HA, McQuillan G, McAllister SK, Fosheim G, et al. Prevalence of Staphylococcus aureus nasal colonization in the United States, 2001-2002. J Infect Dis 2006;193:172-9

4. von Eiff C, Becker K, Machka K, Stammer H, Peters G. Nasal carriage as a source of Staphylococcus aureus bacteremia. Study Group. N Engl J Med 2001;344:11-6

5. Graham PL, 3rd, Lin SX, Larson EL. A U.S. population-based survey of Staphylococcus aureus colonization. Ann Intern Med 2006;144:318-25

6. Kluytmans J, van Belkum A, Verbrugh H. Nasal carriage of Staphylococcus aureus: epidemiology, underlying mechanisms, and associated risks. Clin Microbiol Rev 1997;10:505-20

7. Londoño JF, Ortiz GM, Gaviria AM. Prevalencia de Staphylococcus aureus resistente a meticilina en personal de la unidad de terapia intensiva de la Clínica Universitaria Bolivariana, Medellín 2004. Infectio 2008;10:160-66

8. Bischoff WE, Wallis ML, Tucker KB, Reboussin BA, Sherertz RJ. Staphylococcus aureus nasal carriage in a student community: prevalence, clonal relationships, and risk factors. Infect Control Hosp Epidemiol 2004;25:485-91 
9. Lo WT, Lin WJ, Tseng MH, Lu JJ, Lee SY, Chu ML, et al. Nasal carriage of a single clone of community-acquired methicillin-resistant Staphylococcus aureus among kindergarten attendees in northern Taiwan. BMC Infect Dis 2007;7:51

10. Von Baum H, Schmidt C, Svoboda D, Bock-Hensley O, Wendt C. Risk factors for methicillinresistant Staphylococcus aureus carriage in residents of German nursing homes. Infect Control Hosp Epidemiol 2002;23:511-5

11. Daeschlein G, Assadian O, Rangous I, Kramer A. Risk factors for Staphylococcus aureus nasal carriage in residents of three nursing homes in Germany. J Hosp Infect 2006;63:216-20

12. Lowy FD, Aiello AE, Bhat M, Johnson-Lawrence VD, Lee MH, Burrell E, et al. Staphylococcus aureus Colonization and Infection in New York State Prisons. J Infect Dis 2007;196:911-8

13. Spindel SJ, Strausbaugh LJ, Jacobson C. Infections caused by Staphylococcus aureus in a Veterans'Affairs nursing home care unit: a 5-year experience. Infect Control Hosp Epidemiol 1995; 16:217-23

14. Karabay O, Otkun MT, Yavuz MT, Otkun M. Nasal carriage of methicillin-resistant and methicillinsusceptible Staphylococcus aureus in nursing home residents in Bolu, Turkey. West Indian Med J 2006;55:183-7

15. Koneman EW AS, Janda WM, Schreckenberger PC, Winn WC. Diagnóstico microbiológico. 1999;540-43

16. CLSI. Instituto de Estándares Clínicos y de Laboratorio. Métodos de difusion en discos para determinacion de la sensibilidad a los antimicrobianos. Norma Aprobada, Novena Edición. Documento del Instituto de Estándares Clínicos y de Laboratorio M2-A9 (ISBN 1-56238586-0). 940 West Valley Road, Suite 1400, Wayne, Pennsylvania 19087-1898 USA. Clinical and Laboratory Standards Institute. 2006;

17. Janssens JP. Pneumonia in the elderly (geriatric) population. Curr Opin Pulm Med 2005;11:226-30

18. Bradley SF. Methicillin-resistant Staphylococcus aureus in nursing homes. Epidemiology, prevention and management. Drugs Aging 1997;10:185-98

19. Wendt C, Svoboda D, Schmidt C, Bock-Hensley O, von Baum H. Characteristics that promote transmission of Staphylococcus aureus nursing homes in German nursing homes. Infect Control Hosp Epidemiol 2005;26:816-21

20. Norazah A, Lim VK, Munirah SN, Kamel AG. Staphylococcus aureus carriage in selected communities and their antibiotic susceptibility patterns. Med J Malaysia 2003;58:255-61

21. Loeb MB, Craven S, McGeer AJ, Simor AE, Bradley SF, Low DE, et al. Risk factors for resistance to antimicrobial agents among nursing home residents. Am J Epidemiol 2003;157:40-7

22. Fukuda M, Tanaka H, Kajiwara $Y$, Sugimura T, Oda E, Suenaga $H$, et al. High-risk populations for nasal carriage of methicillin-resistant Staphylococcus aureus. J Infect Chemother 2004;10:189-91

23. O'Sullivan NP, Keane CT. Risk factors for colonization with methicillin-resistant Staphylococcus aureus among nursing home residents. J Hosp Infect 2000;45:206-10

24. Suetens C, Niclaes L, Jans B, Verhaegen J, Schuermans A, Van Eldere J, et al. Determinants of methicillin-resistant Staphylococcus aureus carriage in nursing homes. Age Ageing 2007;36:327-30 